\title{
Wireless Sensing Framework for Long-Term Measurements of Electric Organ Discharge
}

\author{
Michael Harris ${ }^{1}$, Evan Salazar ${ }^{1}$, Robert Güth ${ }^{2}$, Vishal Nawathe ${ }^{3}$, Mahmoud Sharifi ${ }^{1}$, Wei Tang ${ }^{3}$, Satyajayant Misra ${ }^{1}$ \\ 1 Department of Computer Science, New Mexico State University, Las Cruces, NM USA \\ 2 Department of Biology, New Mexico State University, Las Cruces, NM USA \\ 3 Klipsch School of Electrical and Computer Engineering, New Mexico State University, Las Cruces, NM USA \\ $\{$ mharris, sevan, rgueth, vishalsn, msharifi, wtang, misra\}@nmsu.edu
}

\begin{abstract}
This paper describes a customizable/extensible wireless sensor framework for long term monitoring and visualization of the electric organ discharges (EOD) of a weakly electric fish (Sternopygus macrurus). The system developed can be used to simultaneously visualize, in near-real time, the EOD signal of multiple fish residing in separate tanks. Furthermore, the sensing mechanisms used to detect EOD are non-invasive, minimizing side effects on the fishs' natural behavior. Commercial-off-theshelf (COTS) components are used extensively in this framework to lower cost of the system and its future enhancements. COTS components can be easily customized to support different sensors or communication methods. Experimental results involving four fish show accurate measurement of the electric field waveforms and indicate the presence of circadian rhythms in the EOD of the fish.
\end{abstract}

Keywords: Wireless sensor networks, monitoring, data gathering, wireless system, electric fish.

\section{INTRODUCTION}

Technical advances in computer and wireless network technology and recent progress in the accessibility and affordability of these technologies have made it possible to build compact and cost-effective wireless sensor nodes. Such sensors are capable of acquiring large datasets over long periods of time and transmitting them wirelessly to remote access stations for analysis. The need for acquiring long-term data is exemplified by research conducted in areas, such as biology [1], [2] and agriculture [3]. In these areas, long-term data collection by traditional (non-automated) means is time consuming and requires human monitoring, which is expensive and unsustainable.

Long-term automated data acquisition and storage are particularly valuable in research involving live animals due to their temporally dynamic behavior. One such topic is the study of the electric fields produced by electric fish. These fields are studied for their use in navigation, communication, courtship, and, in the case of strongly electric fish such as the electric eel (Electrophorus electricus), for defense and prey capture [4], [5], [6]. The electric fields are created by the synchronized discharge of specialized cells called 'electrocytes,' which make up the fish's electric organ. Long term, automated, and scalable data collection systems are important tools for studying natural EOD variations between individuals as well as variations due to behavioral or environmental factors. Wireless sensor nodes promise greater utility for such long-term data acquisition.

\section{A. Current Solutions}

EOD signals have been previously recorded in live electric fish in order to study the effects of variables including time of day, water temperature, and social interactions on EOD. Parameters such as frequency, waveform, and amplitude have been studied [7], [8], [9], [10], [11]. One popular method of EOD-recording involves the use of a small recording tube carrying metal electrodes. A single fish is then transferred into this tube, which is connected to an oscilloscope or a computer [11]. Because this approach requires handling of the fish, it is not suitable for long-term studies for two main reasons: first, manual handling is labor intensive and practically limits the sampling frequency to once or twice per day. Second, handling of the fish may stress the fish and affect EOD itself.

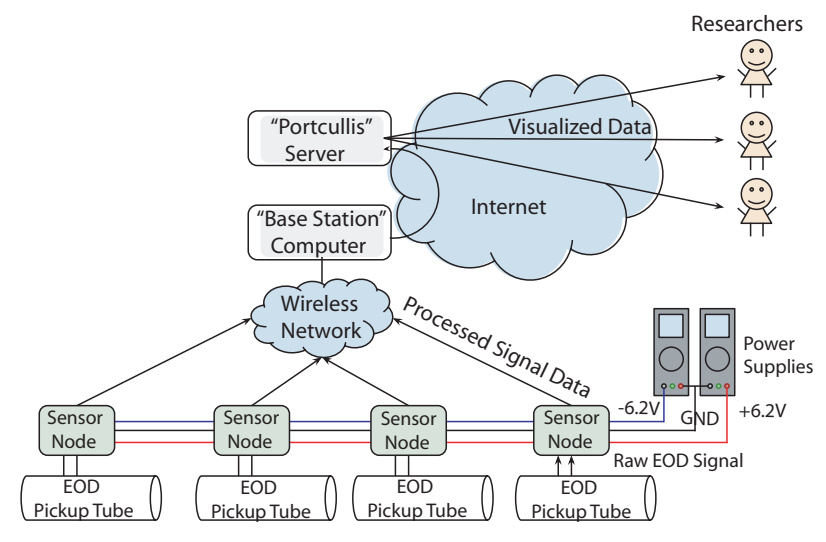

Fig. 1. An overview of our system

Another popular approach has been the use of recording tanks outfitted with electrodes and, in one design, with a centrally located tube outfitted with a detector which starts the EOD-recording process when the fish is inside [7], [8], [9]. In the longest study in the literature, this setup has been used to report EOD-frequency of electric fish once per minute for one month [7]. However, this approach is limited by the requirement of large, specially outfitted recording tanks and has, to our knowledge, not been used to obtain measurements from multiple fish at the same time. In a similar approach, Deng et al [10] used tanks outfitted with wire electrodes connected to a commercial data acquisition system to report EODs from several electric fish simultaneously (reporting once 


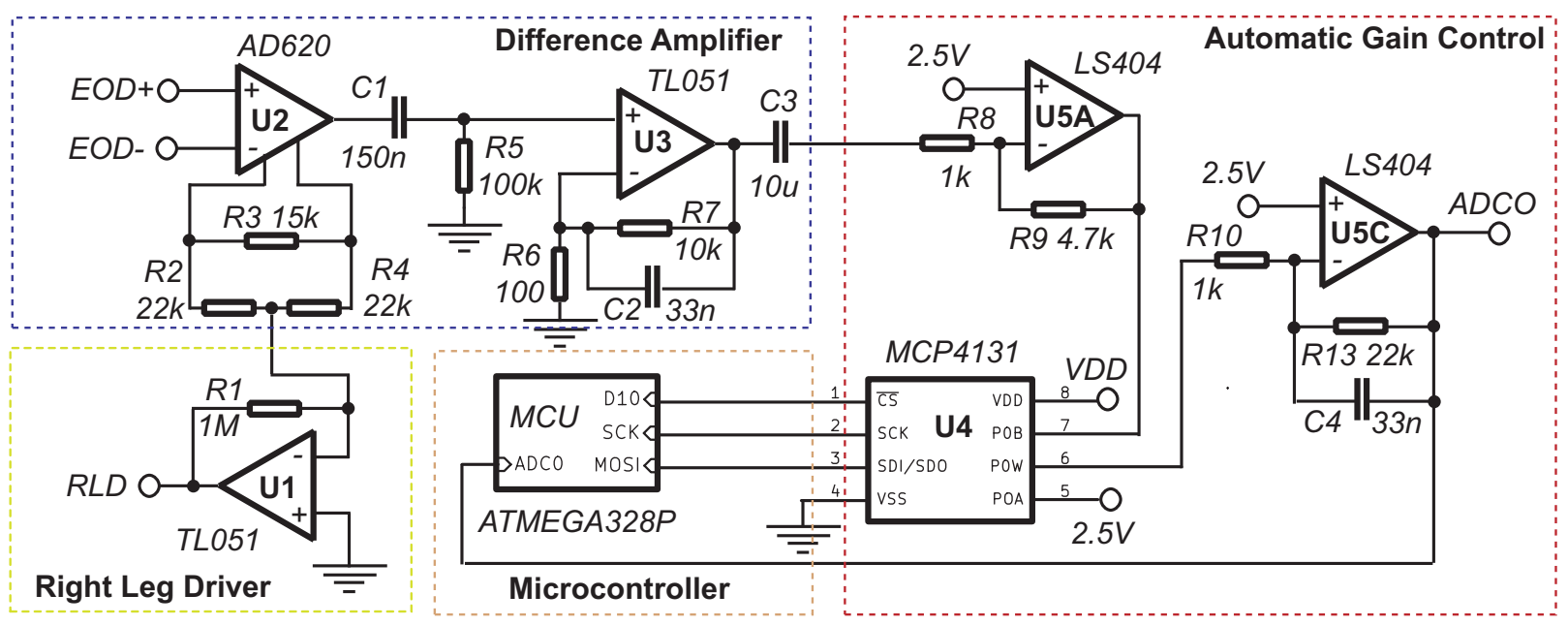

Fig. 2. Schematic of the sensing circuit

every minute for up to 2 weeks). However, this kind of setup requires costly, computer based data acquisition systems and, to our knowledge, has not been used for studies on the scale of months.

\section{B. Contributions of Our System}

In order to perform such long-term experiments, we have developed a wireless sensing framework that allows for the simultaneous recording of EOD-frequencies from multiple fish over long time periods. For our proof-of-concept system (Fig. 1), measurements of EOD-frequency were performed for fish of the species Sternopygus macrurus. The sensing system comprises multiple components including field electrodes placed on plastic tubes, analog amplifiers with automatic gain control, signal processing, wireless data transmission using the IEEE 802.15.4 protocol, data storage and visualization. Data storage and visualization are Internet-based to allow researchers near-real time access to data from anywhere in the world (Link: http://nsoldata.nmsu.edu/portcullis/). Furthermore, the design of the sensor nodes themselves allow for concurrent measurement of up to three more environmental variables per tank (e.g. water temperature, light intensity, water salinity, etc.). The data storage and visualization software is scalable to thousands of data sources, making the framework extensible for future experiments.

\section{System AND Circuit Design}

The framework consists of four main design components: non-invasive electrodes for EOD pickup, signal conditioning and processing, radio communication, and data collection and visualization.

\section{A. Non-Invasive Electrodes for EOD Pickup}

The EOD pickup tubes (Fig. 1) were designed to minimize impact on the fish's environment. To accomplish this, we attached electrodes (EOD+ and EOD- in Fig. 2) to plastic cylindrical tubes. Plastic tubes are a traditional fixture in aquarium habitats for fish such as $S$. macrurus. Our data show that EOD frequency can be recorded over long periods of time with few erroneous measurements (refer Section III). This indicates that the fish remain resting within the tubes for most of the day (22-23 hours/day), a finding that is supported by scientific observations. This makes the arrangement a convenient means for non-invasive electrode placement. Several electrode pickup designs were explored including rings, coils, uninsulated and insulated dipoles, and point electrodes. We also experimented with placement of the electrodes with respect to the fish's body and found the optimal placement to be from the tip of the tail to approximately one-third of the distance between the tip of the tail and the fish's head. These observations concur with previously reported electric field measurements in Eigenmannia virescens, a species closely related to $S$. macrurus with similar electric organ anatomy [12]. Because the fish are unconstrained and vary in size, electrode placement is approximate. Variations in fish size and position are compensated for by signal conditioning circuitry.

\section{B. Signal Conditioning and Processing}

Signal Conditioning: The signal conditioning circuit, shown in Fig. 2, is composed of four components: a differential amplifier for initial amplification, a right leg driver (RLD) for noise cancellation, an automatic gain control (AGC) stage, and a microcontroller (MCU) for data processing and AGC control. As the EOD of the fish is very weak (an estimated $1-2 \mathrm{mV}$ at the electrodes) [4], the analog input of the sensor node is required to have a high gain (around $60 \mathrm{~dB}$ ) to bring it to the operating range $(0-5 \mathrm{~V})$ of the analog to digital converter (ADC) of the MCU. To achieve this, the differential input is first fed to a high impedance instrumentation amplifier (AD620). This amplifier has a fixed gain of $16.9 \mathrm{~dB}$ and a good common-mode rejection ratio. The common-mode of the water is further rejected by attaching a right leg driver (RLD) circuit to the instrumentation amplifier. The output of the AD620 is then fed to a low pass filter with a corner frequency of $10 \mathrm{~Hz}$ which is then fed to a non-inverting amplifier (U3) with a gain of $40.1 \mathrm{~dB}$.

In order to compensate for differences in fish size and the fish's natural movements inside the tubes, the primary amplifier stage is paired with an AGC. The key element of the AGC is a $10 \mathrm{~K}-\mathrm{ohm}$ digital potentiometer (Fig. 2, U4), which sits between two inverting amplifiers, U5A and U5C (Fig. 2). 
These two amplifiers invert the signal and amplify it by $13.4 \mathrm{~dB}$ and $26.8 \mathrm{~dB}$ respectively, centering the voltage at half of $V_{C C}\left(V_{C C}=5 V\right)$. The signal is attenuated by the digital potentiometer up to $-42.1 \mathrm{~dB}$, in 127 discrete steps. This brings the total dynamic amplification capability of the circuit to a minimum of $55.1 \mathrm{~dB}$ and a maximum of $97.2 \mathrm{~dB}$. The MCU samples the maximum amplitude of the signal and controls the digital potentiometer via a serial peripheral interface (SPI) connection. This completes a feedback loop that keeps the signal in range of the ADC and prevents clipping.

Signal Processing: To economize data transmission over the radio link, initial signal processing is done on the sensor node by the ATMega328P, a low-cost 8-bit MCU. The ATMega was chosen in part due to its compatibility with the Arduino development environment [13], which makes it very easy to program. The ATMega328P has a small amount of RAM, so the signal frequency is computed using a simple zerocrossing algorithm. Frequency measurement is done using a microsecond timer that is triggered on a rising edge. The timer runs until 10 total edges are detected, at which point the timer is stopped and the frequency is computed and stored. Every ten seconds, the most recently measured frequency and certain AGC control parameters are transmitted over a radio link for long term storage.

\section{Radio Communication}

The design parameters for the radio link were: high reliability, low power, and, most importantly, availability as a COTS component. The XBee [14] radio satisfied all of these requirements and offered the additional advantage of sharing a socket footprint with several other radio modules (WiFi, Bluetooth, and others). We also considered WiFi, but the available WiFi modules were not found to be particularly reliable, especially on a congested campus network. We found the ZigBee protocol to be highly stable and it provided sufficient bandwidth for our needs. The XBee radio module has a self-contained radio stack, which enables the base station to multiplex data packets received from the sensors. Fig. 3 shows the sensor circuit board with the attached XBee radio.

Because the aquarium facility lacks wired network access, the sensor nodes transmit frequency data to a base station in another room.

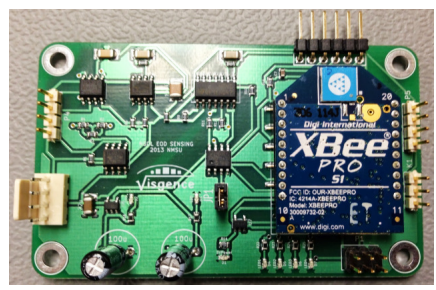

Fig. 3. Our sensor circuit board

\section{Data Collection and Visualization}

The base station consists of a laptop computer connected to an Xbee radio via USB. The computer runs a minimal Linux installation and a simple Python program which listens for incoming data from the sensor boards.
Three pieces of data are collected: maximum ADC value (signal amplitude), the attenuation parameter of AGC, and the characteristic frequency of the EOD signal. The signal frequency is the primary interest of this study. The AGC and amplitude parameters aid in analysis of system integrity. The base station relays this data from the sensor nodes to a server running the Portcullis data collection software [15]. Data is formatted in the Javascript Object Notation (JSON), which the base station encapsulates in HTTP requests. Portcullis (also written in Python) decodes these requests and inserts the data into a SQL database.

The Portcullis system allows users (researchers) to access and visualize data world-wide over the Internet through computer workstations and mobile devices (Fig. 5). The user interface can be configured to continuously update with live readings, zoom-in on a specific time period, filter and aggregate the data through different algorithms, or download raw datasets for custom processing. All these functionalities are important for monitoring of animals and for identifying alterations in EOD frequencies to obtain scientific insights into the biology of electric organ activity.

\section{EXPERIMENTAL RESULTS}

Fig. 4 shows the components of our experimental setup. The experiments took place in a facility housing several dozen interconnected tanks, of which four were instrumented. Sensor nodes were enclosed in PVC electrical junction boxes and hung on the sides of each tank. Because mains power is readily available in the facility, the sensor nodes were powered using low-cost bench power supplies, backed with a small uninterruptible power supply (UPS).

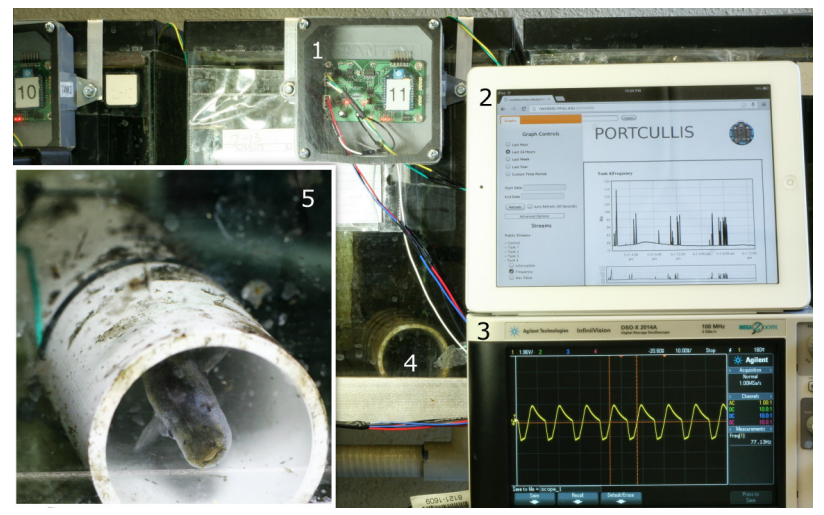

Fig. 4. Components of the experimental setup. Clockwise from top: (1) sensor node, (2) data on the web interface, (3) oscilloscope showing the electric-field waveform, (4) EOD pickup tube with (5) S. macrurus inside.

As a proof of principle, EOD frequency measurements have been obtained from four $S$. macrurus fish simultaneously at a rate of one measurement every 10 seconds, for around six weeks. To verify the accuracy of our system, reported frequency reading from the sensor boards were compared with oscilloscope measurements and an hour-long recording of a raw waveform. The waveform recording was obtained by interfacing the amplifier circuit at ADC0 (Fig. 2) to a laptop computer running Audacity [16] and was analyzed with 
Tank 4|Frequency
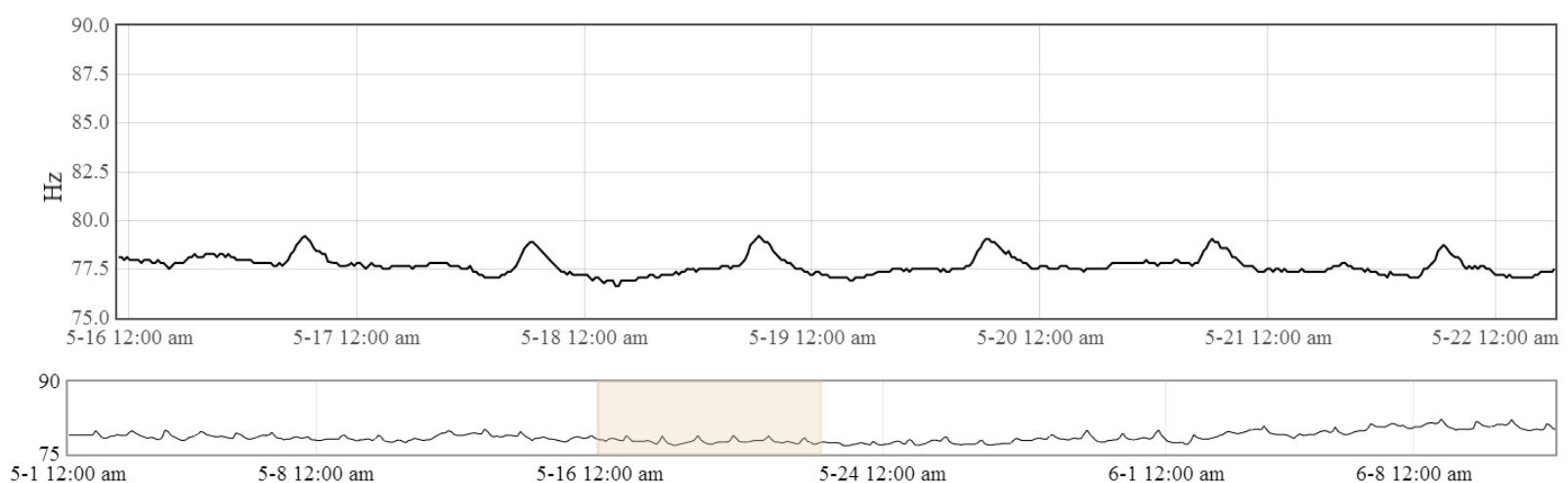

Fig. 5. EOD frequency data (from 5/1/13 to 6/12/13) recorded from one S. macrurus fish and displayed by our web-based interface. The interface shows an overview of the data (bottom) and allows for expansion of areas of interest (highlighted). The expanded section (top) better visualizes the circadian rhythm.

a high resolution FFT (8192 bins). The primary frequency of the FFT closely matched the frequency reported by our sensors. The zero-crossing algorithm results from a sensor board (Tank \#4 in this case) were also compared to frequency values obtained from the raw waveform recording. Frequency data was sampled randomly to obtain 352 sample points for both measurements. The mean difference between the two measurements was less than $0.03(\mu=0.0289)$ with standard error of 0.0065 . With a confidence interval of $95 \%$ the values of the calculated frequencies were within $0.0128 \mathrm{~Hz}$ of the frequencies from raw data.

Fig. 5 shows the frequency measurements of the fish in Tank \#4 gathered over almost six weeks (5/1/2013 - 6/12/2013), we have expanded a small portion from 5/16-5/22. The expanded portion shows diurnal change in the frequency with the frequency peaking around $6: 30 \mathrm{pm}$ daily. According to initial analysis of these results by our biologist collaborators, this diurnal pattern is a strong indication of a circadian rhythm in the EOD of the fish. Occasionally, there may be outlier datapoints in the graphs, resulting from environmental noise or the fish moving outside of its tube. These erroneous readings can be filtered out by statistical methods, for example, by binning the data points into sets and taking the median of each bin as done in related studies [7].

\section{CONCLusions AND Future Work}

A framework to measure EOD frequency of $S$. macrurus over long periods of time (order of months) has been demonstrated. The sensing platform, developed using COTS components and open-source software development tools, provides customizability and expandability at a low cost. The accuracy of the calculated signal frequencies were verified by comparison with raw data. Future work will focus on lowering energy consumption and size of sensor components, enabling the creation of a low-cost implantable sensor for EOD measurement. Like our proposed framework, the implantable sensor will serve as a foundation for future research.

\section{ACKNOWLEDGMENTS}

This research is funded in part by NSF grant CNS-1248109 and NIH grant 1SC1GM092297-01A1. The information re- ported here does not reflect the position or the policy of the federal government.

\section{REFERENCES}

[1] Y. Li, J. Chen, L. Chen, W. Lin, and C. Chu, "Wireless implantable biomicrosystem for bladder pressure monitoring and nerve stimulation," in IEEE BioCAS, 2012, pp. 296-299.

[2] F. Mounaim, G. Lesbros, and M. Sawan, "Long-term monitoring of electrochemical parameters from stimulated neural tissues," in IEEE BioCAS, 2007, pp. 25-28.

[3] Y. Yuan, S. Li, K. Wu, W. Jia, and Y. Peng, "Focus: A cost-effective approach for large-scale crop monitoring with sensor networks," in IEEE International Conference on Mobile Adhoc and Sensor Systems (MASS), 2009, pp. 544-553.

[4] B. Kramer, Electroreception and communication in fishes. Gustav Fischer, 1996, vol. 42.

[5] W. Heiligenberg, "The jamming avoidance response in the weakly electric fish eigenmannia," Naturwissenschaften, vol. 67, no. 10, pp. 499-507, 1980.

[6] A. Mills, H. Zakon, M. Marchaterre, and A. Bass, "Electric organ morphology of sternopygus macrurus, a wave-type, weakly electric fish with a sexually dimorphic eod," Journal of neurobiology, vol. 23, no. 7, pp. 920-932, 1992.

[7] P. Stoddard, M. Markham, V. Salazar, and S. Allee, "Circadian rhythms in electric waveform structure and rate in the electric fish brachyhypopomus pinnicaudatus," Physiology \& Behavior, vol. 90, no. 1, pp. 11-20, 2007.

[8] C. Franchina and P. Stoddard, "Plasticity of the electric organ discharge waveform of the electric fish brachyhypopomus pinnicaudatus i. quantification of day-night changes," Journal of Comparative Physiology A, vol. 183, no. 6, pp. 759-768, 1998.

[9] C. Franchina, V. Salazar, C. Volmar, and P. Stoddard, "Plasticity of the electric organ discharge waveform of male brachyhypopomus pinnicaudatus. ii. social effects," Journal of Comparative Physiology A, vol. 187, no. 1 , pp. $45-52,2001$.

[10] T. Deng and T. Tseng, "Evidence of circadian rhythm of electric discharge in eigenmannia virescens system," Chronobiology International, vol. 17, no. 1, pp. 43-48, 2000.

[11] A. Silva, L. Quintana, M. Galeano, P. Errandonea, and O. Macadar, "Water temperature sensitivity of eod waveform in brachyhypopomus pinnicaudatus," Journal of Comparative Physiology A, vol. 185, no. 2, pp. 187-197, 1999.

[12] E. Knudsen, "Spatial aspects of the electric fields generated by weakly electric fish," Journal of Comparative Physiology, vol. 99, no. 2, pp. 103-118, 1975.

[13] "Arduino development environment," http://arduino.cc/.

[14] "Xbee wireless rf modules," http://www.digi.com/xbee/.

[15] "Portcullis," http://github.com/visgence/portcullis.

[16] "Audacity," http://audacity.sourceforge.net/. 